\title{
Stage IIIA Primary Peritoneal Cancer AJCC v8
}

National Cancer Institute

\section{Source}

National Cancer Institute. Stage IIIA Primary Peritoneal Cancer A/CC v8. NCI Thesaurus. Code C140009.

Stage IIIA includes: T3a, N0/N1, M0. T3a: Primary peritoneal cancer with microscopic extrapelvic (above the pelvic brim) peritoneal involvement with or without positive retroperitoneal lymph nodes. N0: No regional lymph node metastasis. N1: Positive retroperitoneal lymph nodes only (histologically confirmed). M0: No distant metastasis. (AJCC 8th Ed.) 